\title{
Analysis of Puerarin and Chemical Compositions Changes in Kudzu Root during Growth Period
}

\author{
Yiguo Zhao, ${ }^{1,2,3}$ Mei Xu, ${ }^{1,2}$ Zhinneg You, ${ }^{1,2}$ Dongsheng Li, ${ }^{1,2}$ \\ Mingquan Zhou, ${ }^{4}$ Yupeng Zhu, ${ }^{1,2}$ and Chao Wang ${ }^{1,2}$ \\ ${ }^{1}$ Hubei Collaborative Innovation Center of Industrial Fermentation, Wuhan, Hubei 430068, China \\ ${ }^{2}$ Research Center of Food Fermentation Engineering and Technology of Hubei, Wuhan, Hubei 430068, China \\ ${ }^{3}$ Suizhou Eryuefeng Food Co., Ltd., Suizhou, Hubei 441300, China \\ ${ }^{4}$ Lianou Center, Wuhan University, Wuhan, Hubei 430068, China
}

Correspondence should be addressed to Chao Wang; wangchao5412@163.com

Received 28 August 2014; Revised 27 November 2014; Accepted 11 December 2014; Published 25 December 2014

Academic Editor: Huu H. Ngo

Copyright (c) 2014 Yiguo Zhao et al. This is an open access article distributed under the Creative Commons Attribution License, which permits unrestricted use, distribution, and reproduction in any medium, provided the original work is properly cited.

\begin{abstract}
The kudzu root is one of the earliest medicinal plants listed in traditional Chinese medicine. In this paper, chemical compositions changes of kudzu roots from one year old to five years old were analyzed with respect to puerarin, acid-insoluble polysaccharides, acid-soluble polysaccharides, reducing sugar, protein, free amino acids, and lipid. In addition, the puerarin content was determined by high performance liquid chromatography (HPLC) method. The results showed that acid-soluble polysaccharides content of kudzu root increased with each growth period. In contrast, the acid-insoluble polysaccharides decreased significantly. The contents of reducing sugar and puerarin in kudzu root decreased significantly during its growth period. Beyond that, the contents of protein, free amino acids, and lipid in kudzu root ranged from 31.8 to $45.8 \mathrm{~g} / \mathrm{kg}, 2.21$ to $4.33 \mathrm{~g} / \mathrm{kg}$, and 32.2 to $76.9 \mathrm{~g} / \mathrm{kg}$, respectively. The trend of protein content coincided with the total content of free amino acids, in contrast to lipid. This paper provides a set of data and the select of kudzu root for the processing and development of new products of kudzu root.
\end{abstract}

\section{Introduction}

There are 17 recognized types of kudzu root derived from perennial leguminous plants, which originate from China and have been a part of Chinese culture for bimillennium. Lately, it is common that the kudzu root was used to cure various ailments and disorders from $1600 \mathrm{AD}$ to $1867 \mathrm{AD}$ [1]. Winberry and Jones have reported that this kudzu root is first introduced to the United States as an ornamental plant [2].

Due to the fact that the puerarin products belong to healthy organic food, the research of kudzu root is meaningful. Recently, it is known that kudzu root has a lot of nutritional values. And kudzu root contains 14 35\% starch content [3]. Puerarin and daidzein are two major bioactive isoflavones constituents of kudzu root [4]. Most studies have shown that isoflavones can cure hypertension, angina pectoris, cardiovascular diseases, anoxia, and cerebral ischemia and control alcoholism and alcohol abuse [5-7].
It has been experimentally demonstrated that the contents of these active components (puerarin, acid-insoluble polysaccharides, acid-soluble polysaccharides, reducing sugar, protein, free amino acids, and lipid) are the main objective of the kudzu root. But these changes in nutrient affect the application of plants growing period. Acid-soluble polysaccharides and acid-insoluble polysaccharides are mostly starch and fiber, respectively. Starch, fiber, and puerarin are the most important compositions of kudzu root. But little information on the three compositions and other compositions (e.g., free amino acids, lipid, and reducing sugar) is available during the growth period.

It is very necessary to establish the relationship between the kudzu root growth period and nutrition change. To achieve desired product without chemical and physical modification, we will be able to control kudzu-growth period.

This study is to determine the contents of chemical compositions and puerarin, thus finding the trends. These data 
provide a theoretical basis for later study. These trends will provide valuable and meaningful information for both optimal kudzu-growth period and processing operations.

\section{Materials and Methods}

2.1. Materials. Suizhou Eryuefeng Food Co., Ltd., Suizhou City, Hubei Province, China, provided kudzu root (Pueraria lobata). Roots were excavated by shovel method and dried to a constant weight at $70^{\circ} \mathrm{C}$ during December each year. The dry samples were ground into powder for assays and stored in fridge.

Methanol solution is of HPLC grade, from Honeywell International Incorporated (Muskegon, ML, USA). Standard sample of puerarin was bought from the National Institute for the Control of Pharmaceutical and Biological Products, Beijing, China. Ultrapure water was obtained from arium @ 611VF system (Sartorius, Germany) and used throughout the treatments and analyses. The other reagents were A.R.

2.2. Preparation of Standard Curve of Puerarin. The dry sample $(5 \mathrm{~g}$ ) was isolated with $10 \mathrm{~mL}$ ethanol solution (the concentration is $70 \%$ ) at room temperature by ultrasonic wave and the time is $40 \mathrm{~min}$. After the extraction, the extracted slurry was filtered off and the filtrate was collected alone. The filtrate is concentrated and is diluted with ultrapure water to $50 \mathrm{~mL}$. Finally, $1 \mathrm{~mL}$ solution was taken out from $50 \mathrm{~mL}$ solutions and is diluted to $100 \mathrm{~mL}$ solutions with ultrapure water.

2.3. HPLC Analysis of Puerarin Content. Pure standard puerarin is used to establish a standard curve as a positive control. Sample analysis is carried out by reference to the curve. This procedure is performed to the mobile phase, according to the addition of a known concentration of puerarin ranging from 0.00 (control) to $30 \mu \mathrm{g} / \mathrm{mL}$ solutions. The mobile phase without the addition of puerarin is considered as blank group. Due to the addition of various concentrations of puerarin to the mobile phase, the achievements of peaks are used to construct the standard curve, as shown in Figure 1.

The solution is passed through a Millipore filter with the diameter of $0.45 \mu \mathrm{m}$ (Milford, USA) by chromatography analysis method. $10 \mu \mathrm{L}$ solution of the final extract of puerarin is injected into a Shimadzu LGC-1020A equipped with an autosampler, a temperature-controlled column, and a secondary pump. The column is a Dionex C18 $(250 \mathrm{~mm} \times$ $4.6 \mathrm{~mm}, 5 \mu \mathrm{m})$. The mobile phase is carried out with a mixture of methanol and water $(40: 60, \mathrm{v} / \mathrm{v})$ solutions at a flow rate of $1.0 \mathrm{~mL} / \mathrm{min}$ in $25^{\circ} \mathrm{C}$. Data acquisition is detected at a wavelength of $250 \mathrm{~nm}$.

2.4. Determination of Acid-Soluble and Acid-Insoluble Polysaccharides Contents. To determine the acid-soluble polysaccharides content, the dry sample $(2 \mathrm{~g})$ is repeatedly extracted for three times with $15 \mathrm{~mL}$ petroleum ether at room temperature and is centrifuged in $10 \mathrm{~min}$ and the speed is $10000 \mathrm{~g} / \mathrm{min}$. The residue of dry sample is collected and washed again four times with $25 \mathrm{~mL}$ ethanol solution (the

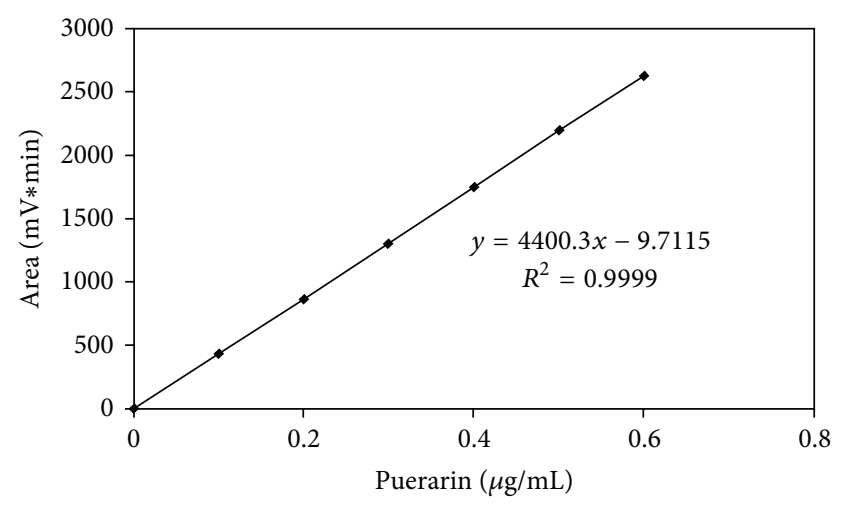

FIGURE 1: Standard puerarin calibration curve. The data were reported as means \pm standard deviation.

concentration is $85 \%$ ), and then it is centrifuged at $10000 \mathrm{~g}$ for $10 \mathrm{~min}$ again. The alcohol-insoluble residue is dissolved with $30 \mathrm{~mL}$ hydrochloric acid solutions (the molar mass is $0.5 \mathrm{M}$ ). The acid-soluble polysaccharides content is estimated by the phenol-sulphuric acid method after being extracted by the filtered hydrochloric acid [8]. Finally, the above-mentioned content multiplied by 0.9 is equal to the acid-soluble polysaccharides content.

To determine the acid-insoluble polysaccharides content, the acid-insoluble residue is collected and oven-dried at $70^{\circ} \mathrm{C}$, and the dry samples is kept in a constant weight state.

\subsection{Detecting the Other Compositions of Kudzu Root}

2.5.1. Determination of Protein. Protein content is determined by the Kjeldahl method (QSY series Kjeldahl apparatus). Firstly, on the condition of the $\mathrm{CuSO}_{4}(0.2 \mathrm{~g}), \mathrm{K}_{2} \mathrm{SO}_{4}$ $(6 \mathrm{~g})$, six glass beads, $0.5 \mathrm{~h}$ time, $240^{\circ} \mathrm{C}$ temperature, the dry sample $(0.2 \mathrm{~g})$ is injected to the $20 \mathrm{~mL}$ sulfuric acid solutions by digestion method. Then the digestion sample is diluted with water to $50 \mathrm{~mL}$, namely, sample solution. Secondly, $10 \mathrm{~mL}$ sample solutions were taken out, and $2 \% \mathrm{H}_{3} \mathrm{BO}_{3}$ solution and $30 \% \mathrm{NaOH}$ solution are added to the $10 \mathrm{~mL}$ sample solutions, and the mix sample solution is titrated to the end point by $0.01 \mathrm{~mol} / \mathrm{L} \mathrm{HCl}$ solution. The calculation formula is as follows:

$$
\text { Protein content }=\frac{c\left(V_{1}-V_{2}\right) \times 0.014}{W} \times \frac{V}{V_{3}} \times F \times 100 \% .
$$

$V_{1}$ is the consumption of standard hydrochloric acid liquid by titrated blank.

$V_{2}$ is the consumption of standard hydrochloric acid liquid by titrated sample solution.

$C$ is the concentration of standard hydrochloric acid liquid.

$V$ is the total volume of sample solution.

$V_{3}$ is the dosage of sample solution.

$W$ is the quality of dry sample.

$F$ is the conversion ratio of protein (6.25). 


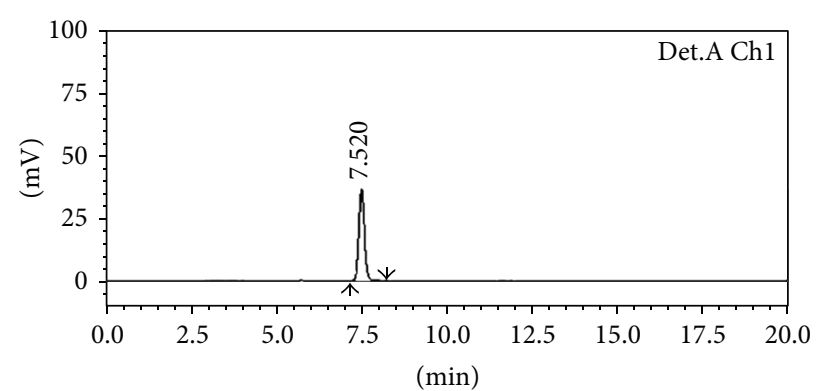

(a)

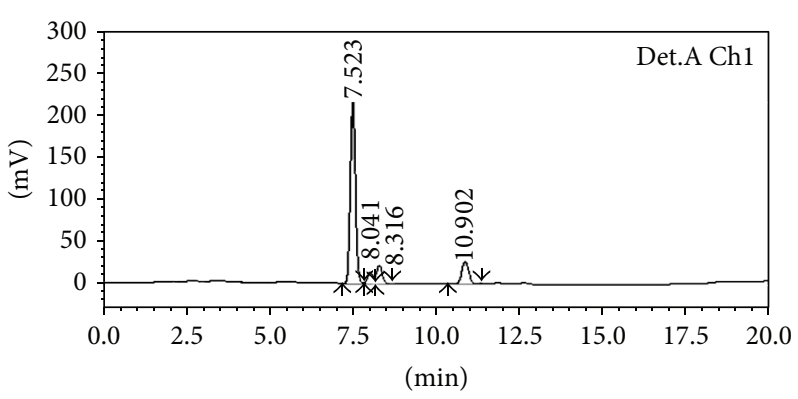

(b)

Figure 2: (a) Chromatograms of puerarin standard by HPLC. The data were reported as means \pm standard deviation. (b) Chromatograms of puerarin sample by HPLC. The data were reported as means \pm standard deviation.

2.5.2. Determination of Free Amino Acids Contents. The dry sample ( $2 \mathrm{~g}$ ) is diluted with $15 \mathrm{~mL}$ trichloroacetic acid solution (the concentration is 3\%). Then the sample is placed at room temperature for $1 \mathrm{~h}$. Above all, the sample is centrifuged in $20 \mathrm{~min}$ and the speed is $10000 \mathrm{~g} / \mathrm{min}$. Finally, the collected supernatant liquid is filtered with Millipore $0.45 \mu \mathrm{m}$ syringe filters (Milford, USA), and its filtrate is loaded onto analyser equipment. Thus, the free amino acids composition is determined with automatic amino acid analyser (Hitachi L-8800, Japan).

2.5.3. Determination of Lipid Content. Lipid content was measured by extraction with petroleum ether in a Soxhlet system [9]. And the dry sample (3 g) is isolated with ultrapure water and is centrifuged in $10 \mathrm{~min}$ (the speed is $5000 \mathrm{~g} / \mathrm{min}$ ). After Soxhlet extraction method, the sample is kept for $2 \mathrm{~h}$ under $100^{\circ} \mathrm{C}$ temperature. The calculation formula is as follows:

$$
\text { Lipid content }=\frac{m_{1}-m_{2}}{m} \times 100 \%
$$

$m_{2}$ is the quality of reservoir bottles and lipid.

$m_{1}$ is the quality of reservoir bottles.

$m$ is the quality of dry sample.

2.5.4. Determination of Reducing Sugar Content. The dry sample $(2 \mathrm{~g})$ is isolated with $40 \mathrm{~mL}$ ultrapure water, and the temperature is $50^{\circ} \mathrm{C}$ and the time is $30 \mathrm{~min}$. After continuously stirring in ultrasound bath, the sample is centrifuged in $10 \mathrm{~min}$ and the speed is $8000 \mathrm{~g} / \mathrm{min}$. Then the supernatant is collected and is diluted with ultrapure water to $50 \mathrm{~mL}$, namely, sample solution. Thus, reducing sugars content of this final sample solution is assayed by the dinitrosalicylic acid method [10].

2.5.5. Statistical Analysis. All tests are performed in triplicate. The results are reported as means \pm standard deviation. The data are calculated with SPSS statistical software (Version 16.0, The Predictive Analytics Company, Chicago, USA).

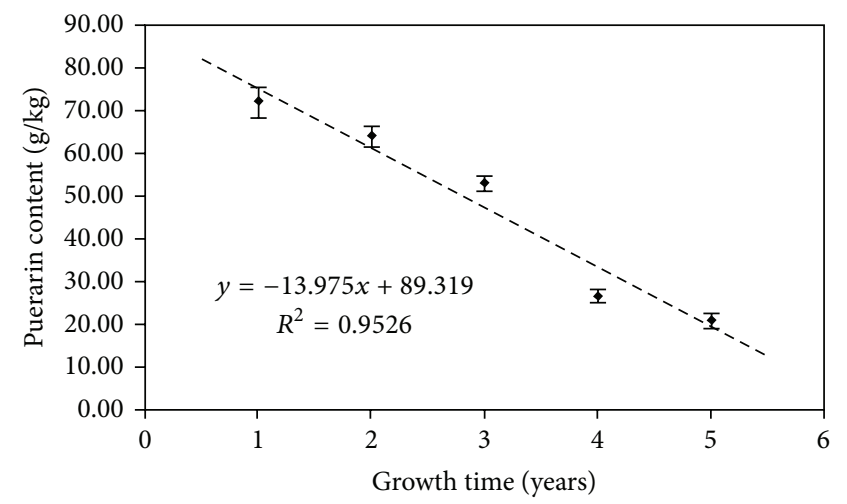

FIGURE 3: Changes of puerarin content of kudzu root during growth. The data were reported as means \pm standard deviation.

\section{Results}

3.1. Changes in Content of Puerarin. The puerarin standard chromatogram of the HPLC analysis is shown in Figure 2(a), and the time of peak of puerarin standard is $7.52 \pm 0.02(n=$ 3 ) min. And comparing with Figures $2(a)$ and 2(b), the puerarin sample is consistent with puerarin standard. Under these conditions, it is clear that puerarin can be detected and the amounts of puerarin content exist in kudzu root during growth period.

Amounts of puerarin content in the same area during growth were determined. In Figure 3, with the growth time increasing, the puerarin content descends linearly. And the content of the puerarin decreases from 72.07 to $20.90 \mathrm{~g} / \mathrm{kg}$. But beyond that, the ratio of puerarin content is decreased to $71 \%$. According to the analysis of molecular structure of puerarin, it might be speculated that the puerarin content has relation with reducing sugar of kudzu root. The difference of the puerarin content of kudzu root might have related to the species-of-origin and other aspects, such as soil fertility and geographical location.

3.2. Changes in Acid-Soluble Polysaccharides and Acid-Insoluble Polysaccharides. The acid-soluble polysaccharides and acid-insoluble polysaccharides compositions of kudzu root 


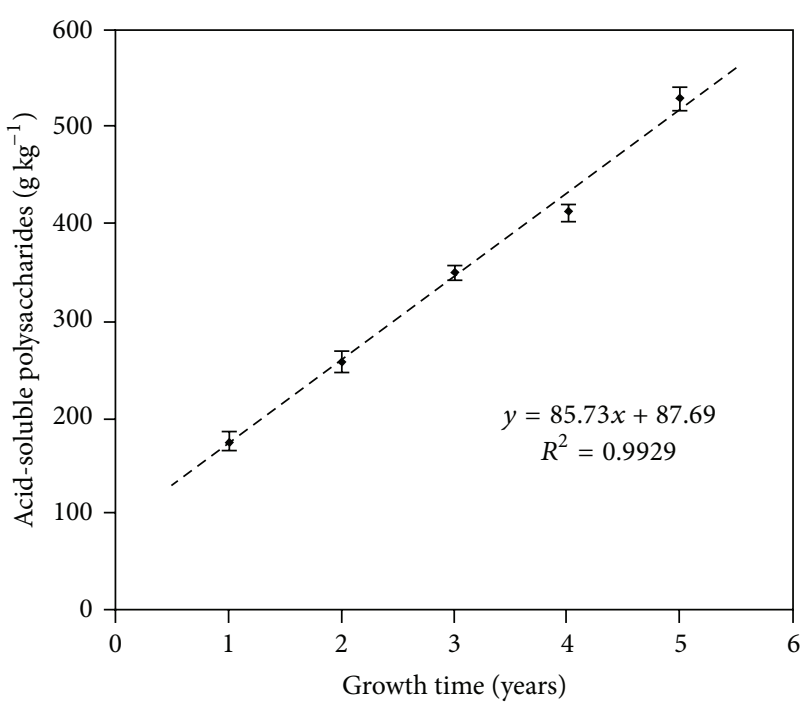

(a)

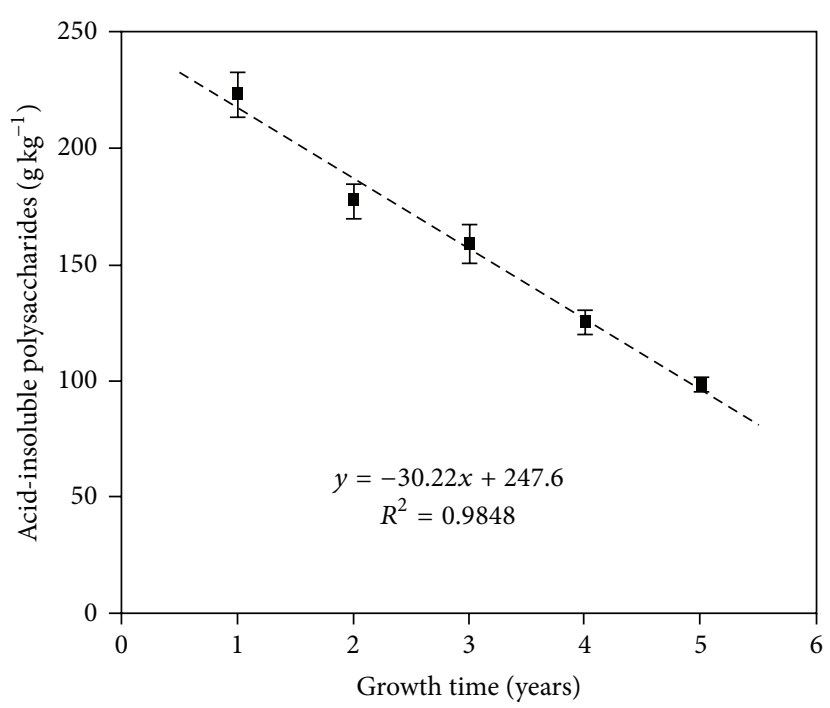

(b)

Figure 4: (a) Changes of acid-soluble polysaccharides contents of kudzu root during growth. (b) Changes of acid-insoluble polysaccharides contents of kudzu root during growth. The data were reported as means \pm standard deviation.

(Pueraria lobata) during its growth are presented in Figures 4(a) and 4(b).

As you can see in Figure 4(a), there is a clear monotonic positive association between acid-soluble polysaccharides contents and growth time of kudzu root. And with the growth time of kudzu root increasing, acid-soluble polysaccharides contents increase from 176.7 to $528.4 \mathrm{~g} / \mathrm{kg}$. It might be speculated that enhanced photosynthesis leads to significant rise in acid-soluble polysaccharides.

Throughout the studied growth period, Figure 4(b) shows that the acid-insoluble polysaccharides content appears to decrease significantly with the increase in the growth time of kudzu root. The acid-insoluble polysaccharides content decreased from 223.8 to $98.8 \mathrm{~g} / \mathrm{kg}$.

According to the total acid-soluble polysaccharides and acid-insoluble polysaccharides, the contents of polysaccharides in kudzu root are increasing during growth period.

3.3. Changes in the Other Chemical Compositions. The other chemical compositions of kudzu root (Pueraria lobata) during its growth are presented in Table 1.

The protein content of kudzu root samples ranges from 31.8 to $45.8 \mathrm{~g} / \mathrm{kg}$. This shows that protein content first increases and then declines along with the increasing of the growth time of kudzu root. And the maximum of protein is $45.8 \mathrm{~g} / \mathrm{kg}$ at the second year. In contrast, the trend of lipid content declines and then increases with the increasing of the growth time of kudzu root, and the minimum is $32.2 \mathrm{~g} / \mathrm{kg}$. Table 1 shows that the reducing sugar content of kudzu root decreases from 99.5 to $38.0 \mathrm{~g} / \mathrm{kg}$ significantly, and the faster reduction of reducing sugar is observed after two years.

The free amino acids composition of kudzu root (Pueraria lobata) during growth is presented in Table 1 . Considerable diversity of amino acids is presented in kudzu root during growth. Kudzu root contains 17 amino acids. All the essential amino acids and half-essential amino acids (threonine, valine, methionine, isoleucine, leucine, phenylalanine, tryptophan, lysine, histidine, and arginine) are detected. Among these amino acids, the content of proline acid (1.21$3.18 \mathrm{~g} / \mathrm{kg})$, alanine acid $(0.15-0.45 \mathrm{~g} / \mathrm{kg})$, and threonine acid $(0.22-0.52 \mathrm{~g} / \mathrm{kg})$ is predominated in kudzu roots during their growth. During the growth period of kudzu roots which are evaluated in this study, the total of free amino acids concentrations appears to increase firstly and then to decline after two years. The trend coincides with the trend of protein content.

\section{Discussion}

The plant's starch content constitutes important indicators for the evaluation of the relative "quality" of plant-derived starch food products $[11,12]$.The puerarin content of kudzu root during the growth period for this study appears to be dissimilar to the previously reported values elsewhere for kudzu root [13]. Kudzu root contains large quantities of isoflavones which belonged to major phytochemicals, and puerarin is the most abundant one, and is an isoflavone C-glycoside [14]. The main composition of acid-soluble polysaccharides is starch. The main composition of acid-insoluble polysaccharides is fiber. Although the content of kudzu root is analyzed, we could not know how to develop good products of kudzu root.

In this paper, we revealed puerarin and other chemical compositions changes in kudzu root (Pueraria lobata) during growth period. The current findings show that the acidsoluble polysaccharides content of kudzu root increased progressively to a significant extent as the growth progressed. The acid-insoluble polysaccharides, reducing sugar, and puerarin contents of kudzu root appeared to decrease with growth time. The significant variability in the content of chemical 
TABLE 1: Changes of chemical compositions of kudzu root during growth ${ }^{\mathrm{a}}$.

\begin{tabular}{lcccc}
\hline Growth time (year) & Protein $^{\mathrm{b}}$ & Free amino acids $^{\mathrm{b}}$ & Lipid $^{\mathrm{b}}$ & Reducing sugar $^{\mathrm{b}}$ \\
\hline 1 & $31.8 \pm 0.05$ & $3.14 \pm 0.09$ & $44.9 \pm 0.17$ & $99.5 \pm 0.25$ \\
2 & $45.8 \pm 0.03$ & $4.33 \pm 0.02$ & $32.2 \pm 0.06$ & $81.5 \pm 0.25$ \\
3 & $37.3 \pm 0.05$ & $3.83 \pm 0.03$ & $54.9 \pm 0.03$ & $56.0 \pm 0.10$ \\
4 & $35.8 \pm 0.07$ & $3.07 \pm 0.02$ & $61.1 \pm 0.07$ & $43.5 \pm 0.35$ \\
5 & $34.1 \pm 0.11$ & $2.21 \pm 0.01$ & $76.9 \pm 0.07$ & $38.0 \pm 0.20$ \\
\hline
\end{tabular}

${ }^{\mathrm{a}}$ The data were reported as means \pm standard deviation, $n=3$.

${ }^{\mathrm{b}}$ All values were expressed as $\mathrm{g} / \mathrm{kg}$ dry weight.

compositions and puerarin of kudzu root during growth provides meaningful and useful information.

According to these results, if we extract starch of kudzu root, we could choose the kudzu that is four and five years old. Dietary fiber plays an important role in keeping the digestive system healthy; we should choose the kudzu that is one year old. Beyond that, we could choose the kudzu in November second year. So, if we can classify and use kudzu root rationally, the resource utilization of kudzu root will reach the maximum.

\section{Conclusion}

The acid-soluble polysaccharides content increases from 176.7 to $528.4 \mathrm{~g} / \mathrm{kg}$ with its growth increasing. In contrast, the acid-insoluble polysaccharides decrease significantly. The content of reducing sugar in kudzu root decreases significantly during its growth. The contents of protein, free amino acids, and lipid in kudzu root range from 31.8 to $45.8 \mathrm{~g} / \mathrm{kg}, 2.21$ to $4.33 \mathrm{~g} / \mathrm{kg}$, and 32.2 to $76.9 \mathrm{~g} / \mathrm{kg}$, respectively. And the total free amino acids coincide with the trend of protein content. The puerarin concentration of kudzu root decreases from 72.07 to $20.90 \mathrm{~g} / \mathrm{kg}$ during the growth process. The above experiments provide a set of data and the select of kudzu root for the processing and development of new products of kudzu root.

\section{Conflict of Interests}

The authors declare that there is no conflict of interests regarding the publication of this paper.

\section{Authors' Contribution}

Yiguo Zhao wrote the paper. Mingquan Zhou interpreted the results. Mei Xu collected the data. Yupeng Zhu and Zhinneg You plotted the figures. Dongsheng $\mathrm{Li}$ and Chao Wang designed the study. Yiguo Zhao and Mei Xu contributed equally to this work.

\section{Acknowledgments}

This study was financially supported by the National Key Technology R\&D Program (2012BAD27B03) and the Scientific and Technological Research and Development Foundation of Hubei Province (YJN0064). The authors gratefully acknowledge the members of the Hubei Food and Fermentation Engineering Technology Research Centre for technical assistance and Suizhou Eryuefeng Food Co., Ltd., for raw material support.

\section{References}

[1] C. C. Bodner and T. Hymowitz, "Ethnobotany of Pueraria species," in Pueraria: The Genus Pueraria, W. M. Keung, Ed., chapter 2, p. 3, Springer, Berlin, Germany, 1986.

[2] J. J. Winberry and D. M. Jones, "Rise and decline of the "miracle vine" Kudzu in the southern landscape," Southeastern Geographer, vol. 13, no. 2, pp. 61-70, 1973.

[3] P. L. Soni and A. Agarwal, "The starch of Pueraria tuberosacomparison with Maize Starch," Starch, vol. 35, no. 1, pp. 4-7, 1983.

[4] M. Miyazawa, K. Sakano, S.-I. Nakamura, and H. Kosaka, "Antimutagenic activity of isoflavone from Pueraria lobata," Journal of Agricultural and Food Chemistry, vol. 49, no. 1, pp. 336-341, 2001.

[5] W. M. Keung and B. L. Vallee, "Kudzu root: an ancient Chinese source of modern antidipsotropic agents," Phytochemistry, vol. 47, no. 4, pp. 499-506, 1998.

[6] H. B. Wong, T. Ma, Y. R. You, and Y. L. Liu, "Effects of daidzein on anoxia and cerebral ischemia in mice," Journal of Shenyang Pharmaceutical University, vol. 16, pp. 63-64, 1999.

[7] Q. L. Zhu and X. R. Lv, "Pharmacology and clinical applications of puerarin," Chinese Traditional and Herbal Drugs, vol. 28, pp. 693-696, 1997.

[8] K. A. C. C. Taylor, "A modification of the phenol/sulfuric acid assay for total carbohydrates giving more comparable absorbances," Applied Biochemistry and Biotechnology, vol. 53, no. 3, pp. 207-214, 1995.

[9] C. S. James, "Determination of fat by the Soxhlet methods," in Analytical Chemistry of Foods, C. S. James, Ed., pp. 91-92, Blackie Academic \& Professional, London, UK, 1995.

[10] T. K. Ghose, "Measurement of cellulase activities," Pure and Applied Chemistry, vol. 59, no. 2, pp. 257-268, 1987.

[11] G. Lisinska and W. Leszczynski, "Chemical composition of tubers," in Potato Science and Technology, pp. 16-43, Elsevier Applied Science, London, UK, 1989.

[12] G. Lisinska and W. Leszczynski, "Potato chips," in Potato Science and Technology, pp. 166-233, Elsevier Applied Science, London, UK, 1989. 
[13] D.-L. Wang, X.-C. Zhang, and Y. Sun, "Study on dynamic accumulation of main components in Pueraria lobata (Willd) Ohwi," Chinese Pharmaceutical Journal, vol. 43, no. 13, pp. 974-977, 2008.

[14] H. Rong, D. D. Keukeleire, L. D. Cooman, W. R. G. Baeyens, and G. V. D. Weken, "Narrow-bore HPLC analysis of isoflavonoid aglycones and their O- and C-glycosides from Pueraria lobata," Biomedical Chromatography, vol. 12, no. 3, pp. 170-171, 1998. 

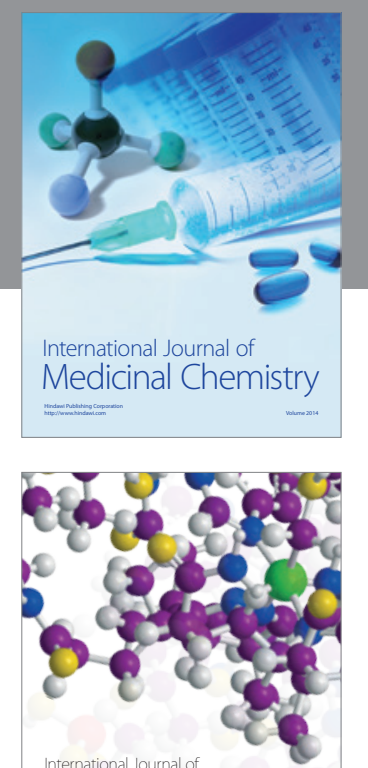

\section{Carbohydrate} Chemistry

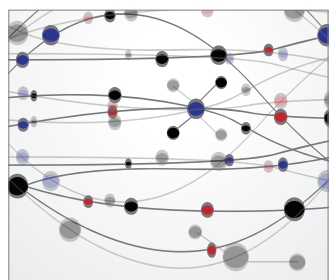

The Scientific World Journal
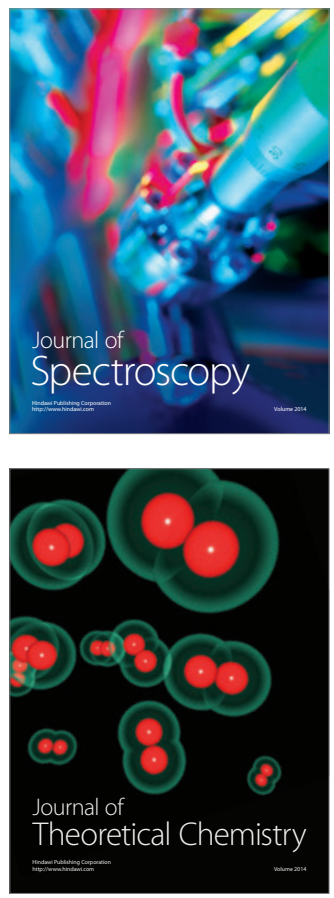
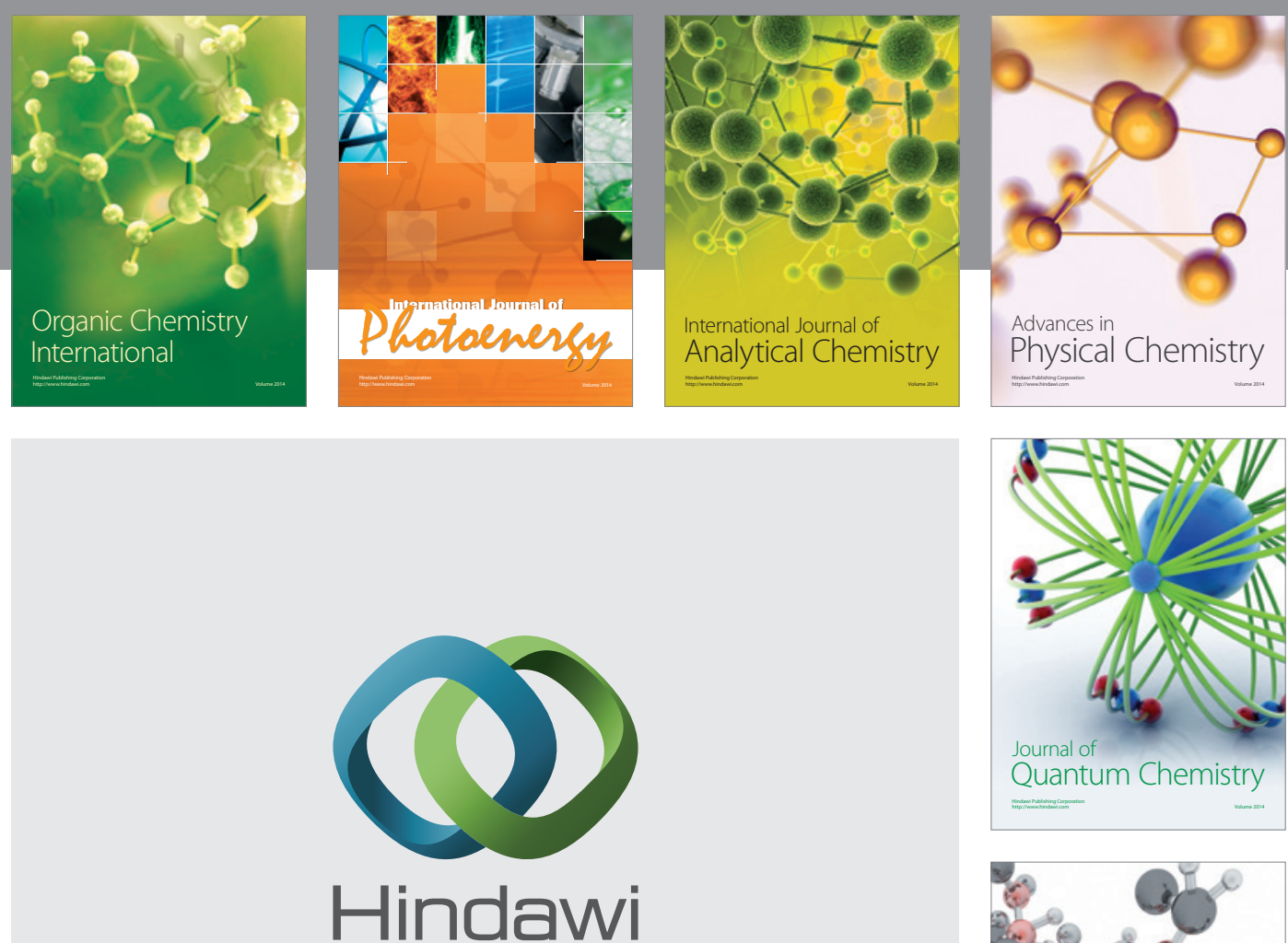

Submit your manuscripts at

http://www.hindawi.com

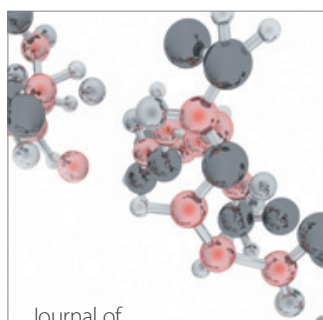

Analytical Methods

in Chemistry

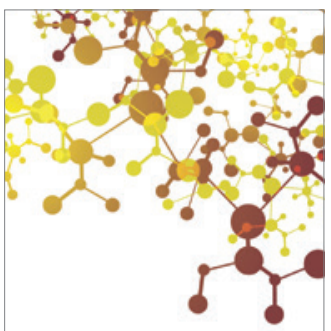

Journal of

Applied Chemistry

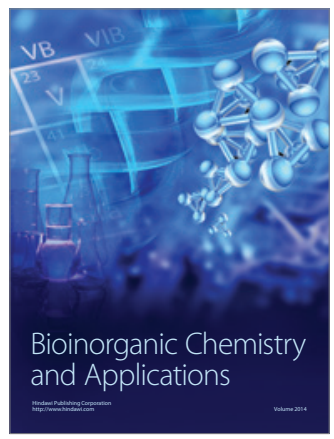

Inorganic Chemistry
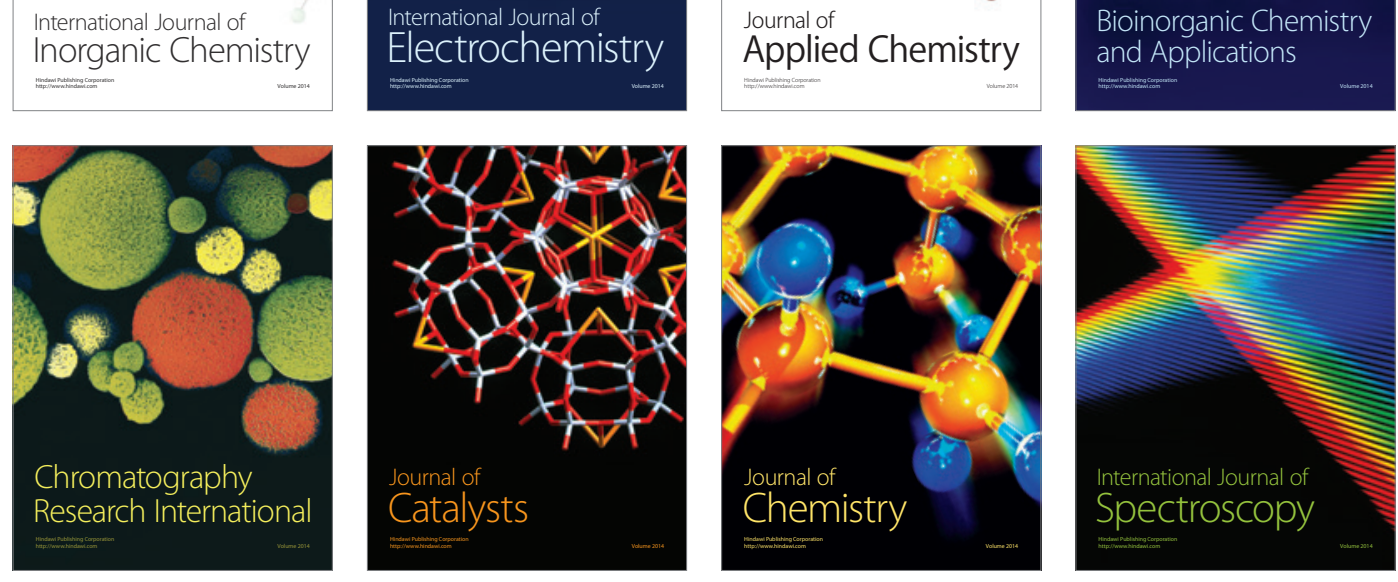